\title{
Development and Aging
}

\section{Attachment-related expectations and mother-referent memory bias in middle childhood}

\author{
ADINDA DUJARDIN, ${ }^{1}$ GUY BOSMANS, ${ }^{1}$ CAROLINE BRAET ${ }^{2}$ and LIEN GOOSSENS ${ }^{2}$ \\ ${ }^{1}$ Parenting and Special Education Research Unit, University of Leuven, Belgium, \\ ${ }^{2}$ Department of Developmental, Personality, and Social Psychology, Ghent University, Belgium
}

\begin{abstract}
Dujardin, A., Bosmans, G., Braet, C. \& Goossens, L. (2014). Attachment-related expectations and mother-referent memory bias in middle childhood. Scandinavian Journal of Psychology.

Understanding the cognitive processing of attachment-relevant information has become a major focus of attachment research. Previous research demonstrated links between attachment and memory for attachment-related information, but results were contradictory and did not control for mood-effects. The current study aimed to provide a conceptual framework to capture inconsistencies. A straightforward memory bias hypothesis was derived and tested. Fifty children (aged 10-12) completed questionnaires assessing confidence in maternal support and depressive symptoms, and a memory task in which they recalled positive and negative words that referred to previous interactions with mother. Less confidence in maternal support and more depressive symptoms were linked to a more negative mother-related memory bias. The effect of confidence in maternal support remained marginally significant when controlling for depressive symptoms, explaining the initial effect of depressive symptoms. These findings support attachment theory's hypothesis that attachment-relevant information is processed in an attachment expectation-congruent way.
\end{abstract}

Key words: Attachment, memory, middle childhood.

Adinda Dujardin, Parenting and Special Education Research Unit, KU Leuven, Leopold Vanderkelenstraat 32, box 3765, 3000 Leuven, Belgium. Tel: +(32)(0)16/326068; e-mail: adinda.dujardin@ppw.kuleuven.be

\section{INTRODUCTION}

Attachment theory states that children who have repeated experiences with responsive parenting develop the expectation that their parents will be available and responsive in times of need. This expectation reflects these children's confidence about attachment figures' availability, which Ainsworth (1973) considered central to secure attachment. Conversely, children who frequently experienced their parents as unresponsive develop uncertainty about parental support, and become insecurely attached (e.g., Ainsworth, 1973; Bowlby, 1969; Bowlby, 1973). Bowlby (1969) stated that these attachment experiences become internalized in a cognitive structure, the internal working model (IWM) of attachment. This IWM's function is thought to be that of storing attachment-related knowledge, and subsequently influencing the way in which people process and operate on attachment-relevant social information (Bowlby, 1973). This implies that attachment-related memories are thought to be crucial for the construction as well as the consolidation of attachment expectations (Collins \& Read, 1994). Bowlby (e.g., 1973) hypothesized that children's memories would be biased in congruence with their attachment expectations. This attachment expectation-congruent memory bias results from two related processes. First, these memories are thought to reflect secure or insecurely attached children's learning history of, respectively, more frequent positive or negative interactions with caregivers. Second, attachment expectations are thought to bias children's memories in the sense that they deplete their ability to recall information that is incongruent with these expectations. Thus, according to Bowlby's (1973) hypothesis, children who are uncertain about caregivers' support will more likely recall attachment-relevant information in a negatively biased way, whereas children who are confident about caregivers' support will more likely recall it in a positively biased way. Previous research found some evidence that attachment-related expectations are associated with children's memory for attachment-relevant information regarding mother (e.g. Alexander, O'Hara, Bortfeld, Anderson, Newton \& Kraft, 2010; Kirsh \& Cassidy, 1997; Lynch \& Cicchetti, 1998; Beinstein Miller \& Noirot, 1999; Rudolph, Hammen \& Burge, 1995). However, the results are often mixed and seem occasionally contradictory (for a review see Dykas \& Cassidy, 2011). Therefore, the current study aimed to investigate memory-processing of attachment-related information based on a clear theoretical perspective on memory biasresearch inconsistencies.

\section{ATTACHMENT AND THE RECOLLECTION OF ATTACHMENT-RELATED INFORMATION}

Contrary to Bowlby's (1973) hypothesis, some studies found evidence suggesting that more securely attached children are biased towards recalling more negative attachment-related information. During these studies, children are typically asked to recall stories regarding interactions between a hypothetical mother-child pair. For example, Alexander and colleagues (2010) found that more securely attached children (7-12 years), as measured with the Security Scale (Kerns, Aspelmeier, Gentzler \& Grabill, 2001), had better recall performance for negative (separation), but not for positive (reunion) attachmentrelated stories. While Kirsh and Cassidy (1997) found better 
recollection for negative (rejecting), but also for positive (responsive) attachment-related stories in children (3-4 years) with a secure attachment classification, as measured with the Strange Situation Procedure (Ainsworth, Blehar, Waters \& Wall, 1978).

Another study, however, might be interpreted as supporting Bowlby's (1973) hypothesis (Rudolph et al., 1995). In this study, mother-related memory bias was measured using a mother-referent encoding task. In this task, children first had to determine whether or not a series of positive and negative adjectives referred to actual interactions with their mother, and, subsequently, had to recall as many of these adjectives as possible. Adjectives that were endorsed as referring to interactions with mother were called mother-referent. Results showed that children (10-12 years) with more negative perceptions of maternal acceptance, responsiveness, and support, as measured with respectively the Acceptance subscale of the Child's Report of Parental Behavior Inventory-Revised (CRPBI; Margolies \& Weintraub, 1977), the Children's Expectations of Social Behavior Questionnaire (CESBQ; Rudolph et al., 1995), and the Social Support Appraisals Scale (APP; Dubow \& Ullman, 1989), recalled a larger proportion of negative versus positive mother-referent words. Although this study provided promising information in light of Bowlby's (1973) hypothesis, responsive parenting is not synonymous for secure attachment (van Ijzendoorn, 1995). This implies that a more direct test of Bowlby's hypothesis using the motherreferent encoding task is warranted.

\section{A THEORETICAL FRAMEWORK FOR UNDERSTANDING MEMORY BIAS RESEARCH INCONSISTENCIES}

The abovementioned inconsistencies might be at least partly explained by paradigmatic differences in the nature of the presented information. While the studies contradicting Bowlby's (1973) hypothesis use hypothetical attachment stories, Rudolph and colleagues (1995) studied the recollection of autobiographically relevant attachment information. One could argue that recalling hypothetical attachment information, typically used in story tasks, differs from recalling autobiographical attachment information (see also Rudolph et al., 1995). Recollection of hypothetical stories depends on the encoding of both attachment expectation-congruent and -incongruent information. Because the latter more strongly attracts attention while being encoded (Johnson, Dweck \& Chen, 2007), the hypothetical stories paradigm might find better recollection for expectation-incongruent than expectation-congruent information. Confirming this assumption, memory bias research suggests that unexpected information often gets better recalled (see Stangor \& McMillan, 1992).

Apart from this, one might wonder whether asking children to recall hypothetical information is the most suitable paradigm for testing Bowlby's (1973) hypothesis, because this hypothesis is based on the recollection of autobiographical attachment information. Moreover, the outcome of this type of memory bias research might be difficult to interpret. Indeed, research suggests that irrelevant and inconsistent unexpected information should be distinguished, because schema-inconsistent information is thought to attract attention and lead to a better recall performance, while this is not the case for schema-irrelevant information (see e.g., Brewer \& Nakamura, 1984; Davidson, 1994).
Because it is difficult to determine which unexpected stories are inconsistent with or irrelevant for children's attachment schema, it is hard to infer strong predictions about the direction of effects when using hypothetical stories. Confirming that recalling hypothetical attachment information does not lead to unidirectional effects, Alexander and colleagues (2010) found better recollection in securely attached children for expectationincongruent stories, while Kirsh and Cassidy (1997) found the opposite. Because of this paradigmatic limitation, merely asking to recall hypothetical stories might not provide a sufficiently straightforward test of Bowlby's hypothesis.

In contrast, recalling autobiographical attachment information cannot be affected by (un)expectedness or (ir)relevance of the encoded information, but should straightforwardly reflect the accessibility of the recollections that nurture expectations. This should lead to expectation-congruent memory biases. In line with this assumption, a plethora of depression studies demonstrated that depression is linked with recollections of less positive autobiographical information (see Wisco, 2009). Consequently, it seems reasonable to assume that the paradigm used by Rudolph and colleagues (1995) might be more useful for studying attachment-related memory biases.

Seemingly contradicting this stance, a study (Lynch \& Cicchetti, 1998) that used Rudolph and colleagues' (1995) memory task reported an attachment expectation-congruent memory bias in children that scored high on victimization as well as in non-maltreated children, and an attachment expectation-incongruent bias in low-victimized and in maltreated children. However, in this study, memory bias scores were calculated based on words that were not endorsed as mother-referent, which implies that this study's memory bias score - as is the case with the hypothetical stories paradigm - does not reflect autobiographically relevant attachment information. Moreover, the results might reflect (severely) traumatized children's coping with relational trauma rather than basic memory processes. Indeed, asking children to recall traumatic information (about interactions with their maltreating mother) might cause severe psychological pain, activating attachment-related defensive exclusion processes, suppressing the memory for negative attachment-related information (e.g., Bowlby, 1973; Dykas \& Cassidy, 2011). Although it is beyond doubt important to study attachment processes in at risk populations, the complex pattern of results suggests that studies aimed at unraveling basic attachment-related memory processes should be carried out in general population samples.

\section{THE CURRENT STUDY}

Given the inconsistent results in previous tests of Bowlby's (1973) attachment expectation-congruent memory bias-hypothesis, the current study was designed to provide an ameliorated test of this hypothesis. Based on the abovementioned analysis of previous research, the current study tested Bowlby's (1973) hypothesis in a general population sample, using only mother-referent words, and a widely used and well-validated measure of perceived confidence in maternal support.

Furthermore, research has shown that children with higher levels of depressive symptoms tend to recall a larger proportion of negative versus positive self-referent and mother-referent 
information (e.g., Hammen \& Zupan, 1984; Neshat-Doost, Taghavi, Moradi, Yule \& Dalgleish, 1998; Timbremont \& Braet, 2004). Therefore, in the current study depressive symptoms was added as a control variable, to rule out the possibility that the link between attachment and mother-related memory bias merely reflects a depression-related effect.

In sum, this study aimed to investigate the link between attachment expectations and memory for mother-related information, hereby controlling for depressive symptoms, using a theoretically appropriate memory bias task, and a well-validated measure of attachment-related expectations, in a general population sample. In line with Bowlby's (1973) hypothesis, it was expected (1) to find an expectation-congruent association between attachment and mother-related memory bias, and (2) that this link would remain present after controlling for depressive symptoms.

\section{METHOD}

\section{Participants}

Fifty children (18 boys, 32 girls) participated in the study, with ages ranging from 10 to $12(M=10.9, S D=0.50), 28 \%$ of which had divorced parents. All children lived with their biological mother, and had been primarily raised by their mother $(42.9 \%)$ or by both parents $(57.1 \%)$ during the first three years of their life. Regarding parental level of education, $2 \%$ of the mothers had an elementary school degree, $44.9 \%$ a high school degree, $36.7 \%$ a post-high school technical training or technical bachelor degree, and $16.3 \%$ a university master degree. With regard to the fathers, $41.6 \%$ had a high school degree, $37.5 \%$ a post-high school technical training or technical bachelor degree, and 20.8\% a university master degree.

\section{Procedure}

Participants were invited to participate in a broader study on cognitive vulnerability for psychopathology in children, using a letter distributed in elementary schools in Flanders. The letter informed about the content of the study, and asked the parents' approval to participate. Approximately $24 \%$ of the parents who received a letter gave their informed consent. Fifty children were randomly selected to participate in the study. All invited children and parents chose to participate after being personally informed about the content and methodology of the study, and about their right to refuse participation. In light of the rather small response rate $(24 \%)$, and the uneven distribution of boys (36\%) and girls $(64 \%)$, Trust scores of the current sample $(M=34.86, S D=4.15)$ were compared to Trust scores $(M=35.16, S D=4.98)$ of a representative sample of 289 Flemish children aged $10-12$ years old (53,3\% boys, $46,7 \%$ girls) who participated in a classroom questionnaire study (Bosmans, Taghavi, Moradi, Yule \& Dalgleish, 2008). Results of an independent samples t-test revealed no significant difference between the Trust scores of both samples $(t(337)=0.40, p=0.69)$. This study was part of a more elaborate two hour test procedure consisting of several questionnaires, followed by an interview and the memory task. Children were visited at home by trained bachelor psychology students. Afterwards participants received a small present to thank them for their participation. The study was approved by Ghent University's ethical committee.

\section{Measures}

Confidence in maternal support. Confidence in maternal support was measured using a Dutch version of the Trust-subscale of the People In My Life Questionnaire (PIML; Ridenour, Greenberg \& Cook, 2006; Bosmans, Braet, Koster \& De Raedt, 2009). The PIML is designed to measure 10 to 12-year-old children's representations of attachment figures. It is a child-friendly version of the widely used Inventory of Parent and Peer Attachment (IPPA, Armsden \& Greenberg, 1987) (e.g., Allen, Porter, McFarland, McElhaney \& Marsh, 2007). This measure has good psychometrics, and good validity in work with adolescents (Allen, in press). Its validity is demonstrated for example through links with attachment figure utilization, self-esteem, and parenting quality (e.g., Armsden \& Greenberg, 1987; Gullone \& Robinson, 2005). In previous research (Ridenour et al., 2006), the PIML has shown to be a valid attachment measure in middle childhood with a factor structure and correlations with measures of symptomology and well-being comparable to the IPPA. For the current study, only the items of the Trust-scale focusing on mother were used. Trust is conceptualized as the positive affective/cognitive experiences of trust in the accessibility and responsiveness of the attachment figure (10 items; e.g., "I can count on my mother to help me when I have a problem'"; Armsden \& Greenberg, 1987). Participants responded on a four-point Likert-scale ranging from 1 (almost never true) to 4 (almost always true). The validity of the Trust scale has been established in several studies. Ridenour and colleagues (2006) for instance showed that it has good internal consistency, and correlated negatively with measures of internalizing and externalizing problems. In a sample of Flemish children, the Trust scale has also been found to have good internal consistency, and to be linked with measures of psychopathology symptoms and parenting styles (Bosmans et al., 2008). Moreover, the scale was previously linked with maternal parenting behaviors (Bosmans, Braet, Beyers, Van Leeuwen \& Van Vlierberghe, 2011) and the breadth of the attentional field around mother (Bosmans et al., 2009), and an observational study has shown that distressed children more quickly seek their mother's proximity when they score higher on the Trust scale (Bosmans, Braet, De Coene. \& De Raedt, 2013). In the current sample the Trust scale $(\alpha=0.84)$ was found to have good reliability.

Depressive Symptoms. To measure depressive symptoms, children completed a Dutch version of the Child Depression Inventory (CDI; Kovacs, 1992; Timbremont \& Braet, 2002). The CDI is used for children and adolescents aged 7 to 17 . It includes 27 items measuring cognitive, affective and behavioral symptoms of depression. Each item consists of three statements graded in order of increasing severity from 0 to 2 , and children select the item that characterized them best during the past two weeks. The item scores are combined into a total depressive symptoms score. The original questionnaire has relatively high levels of internal consistency, test-retest reliability and predictive, convergent and construct validity, especially in non-clinical populations (Craighead, Smucker, Craighead \& Ilardi, 1998). Psychometric results for the Dutch version are in line with the original CDI. In the current sample we found the CDI to have good reliability ( $\alpha=0.83$ ).

Memory Task. To measure memory processing, Rudolph and colleagues (1995) mother-referent encoding task was used, which is a performancebased measure for assessing cognitive processes regarding mother, based on Hammen and Zupan's (1984) widely used self-referent encoding task. This task consists of a rating task (see Mother-Referent Encoding Task), followed by a recall task (see Recall Task).

Mother-Referent Encoding Task. In this task participants had to decide whether a set of positive and negative adjectives refer to emotions experienced during interactions with mother ('Yes'-rated) or not ('No'-rated) (Have you ever felt this way when being together with your mother?). In order to select the emotion words, Dutch-speaking elementary school children, aged 10 to 12 , were asked to construct a short essay about a time when they felt loved/not loved by their mother. Based on these essays, the experimenter selected 44 different Dutch emotion words; 21 positive words (e.g., peaceful, pleasant, agreeable...) and 23 negative words (e.g., angry, scared, guilty ...). The first two words and the last two words (three negative and one positive word) were used as fillers to avoid recency and primacy effects in the recall task (Timbremont \& Braet, 2004). Consequently, 20 negative and 20 positive words were used in the analyses. To control for effects due to word order, two versions of the task were constructed. In both versions the emotion words, 
except for the fillers, were presented in a different order. Children were told to pay attention to the words because afterwards they would be asked to recall as many words as possible. This instruction for intentional recall was given to avoid floor effects (Neshat-Doost et al., 1998). Contrary to the original procedure, however, we did not include structural encoding as a control condition (in which participants are for example asked whether or not it is a long word), because previous research showed that neither attachment, nor depression effects were found in structural encoding conditions (e.g., Lynch \& Cicchetti, 1998; Rudolph et al., 1995; Timbremont \& Braet, 2005). An Encoding Valence score was calculated as the number of positive minus the number of negative words that were endorsed as mother-referent, with higher scores indicating a more positive and lower scores a more negative view of mother.

Recall Task. After the encoding task, the subscale 'mazes' from the Wechsler Intelligence Scale for Children-Revised (WISC-R) was administered for one minute as an additional control for recency effects. Next, the participants were instructed to write down as many words as they could remember from the rating task for two minutes. Similar to previous research (Hammen \& Zupan, 1984; Taylor \& Ingram, 1999), Memory Bias was assessed by examining the percentage of words that the children endorsed as mother-referent in the encoding task ('Yes'-rated words) and that were later recalled. First, a proportional score was calculated for negative and positive words separately. For example, the proportional score for positive words was computed by dividing the number of positive mother-endorsed words that the child recalled by the total number of positive mother-endorsed words. Next, a memory valence score was calculated as the proportion of positive minus the proportion of negative recalled mother-referent words, with higher scores indicating a more positive, and lower scores a more negative memory bias. Research on recall performance typically prefers proportional scores over raw means. Furthermore, 'Yes'-rated words are preferred over 'No'-rated words because the first generally lead to better recall-performance than the latter (Hammen \& Zupan, 1984; Timbremont \& Braet, 2004).

Control variables. Finally, participants were asked to rate each adjective on familiarity, valence, and relevance. Familiarity was assessed by asking the participant whether they knew the words or not. To check whether the participants actually perceived the words as positive or negative, children rated the valence of each word on a seven-point rating scale, ranging from (1) 'very unpleasant' to (7) 'very pleasant'. Finally, to test for relevance, participants rated each word on a seven-point rating scale, ranging from (1) 'never' to (7) 'all the time', as an answer to the question how often they feel this way while being together with their mom.

\section{RESULTS}

\section{Preliminary analyses}

There were no missing data, except for the questions regarding familiarity, valence and relevance of the words, which were missing for one child that had to leave sooner. Trust was significantly negatively correlated with Depressive symptoms $(r(48)=-0.31, p=0.03)$. Gender was significantly related to Trust $(F(1,48)=6.27, p=0.02)$, with higher scores for girls $(M=35.91, S D=3.40)$ compared to boys $(M=33.00, S D=$ 4.77). Gender was not significantly related to Depressive symptoms. Neither Trust nor Depressive symptoms correlated significantly with age. There was no effect of age $(F(1,48)=1.29$, $p=0.26)$ or gender $(F(1,48)=0.07, p=0.79)$ on the Encoding Valence score, and both age $(F(1,48)=0.01, p=0.91)$ and gen$\operatorname{der}(F(1,48)=2.17, p=0.15)$ also did not have an effect on the Memory Valence score. Mean and standard deviations for Trust and Depressive symptoms were $(M=34.86, S D=4.15)$, and $(M=7.86, S D=5.57)$ respectively.

With regard to the memory task's control variables, results showed that scores for familiarity varied between $83.7 \%$ and $100 \%$, except for the word 'disadvantaged,' that was only known by $49 \%$ of the participants. Concerning the valence, mean pleasantness scores for all positive words were situated on the positive side of the scale (ranging from $M=5.92$ for the word 'restful' to $M=6.76$ for the word 'happy'), and mean pleasantness scores for all negative words were situated in the negative side of the scale (ranging from $M=1.29$ for the word 'unhappy' to $M=3.18$ for the word 'lazy'). Finally, the mean scores for relevance ranged from $M=2.08$ for the words 'bad' and 'failed' to $M=6.24$ for the word 'safe', indicating that none of the words was generally perceived as irrelevant for the relationship with mother.

On average children endorsed $86 \%(S D=0.14)$ of the positive, and $48 \%$ ( $S D=0.26)$ of the negative words as motherreferent in the encoding task. The result of a one-sample t-test on the Encoding Valence scores $(M=0.38, S D=0.26)$ was strongly significant $(t(49)=10.39, p<0.001)$, indicating an overall positive view of mother. Additionally, to investigate the link between Trust and Encoding Valence, Pearson's correlation was calculated. Results revealed that this correlation was significantly positive $(r(48)=0.43, p=0.002)$, indicating that higher Trust scores are related to a more positive view of mother.

\section{Overall valence of recall}

On average, participants recalled $17 \%(S D=0.10)$ of the positive, and $13 \%(S D=0.10)$ of the negative words that were endorsed as mother-referent. The result of a one-sample t-test on the Memory Valence scores $(M=0.07, S D=0.11)$ was strongly significant $(t(49)=4.49, p<0.001)$, indicating an overall positive mother-referent Memory Bias.

\section{Trust, depressive symptoms and memory bias}

To investigate the effect of Trust and Depressive symptoms on children's memory valence for mother-related information, a series of correlational analyses was conducted. First, Pearson's correlation between Trust and Memory Valence was significantly positive $(r(48)=0.34, p=0.01)$, indicating that lower Trustscores are associated with a more negative mother-related Memory Bias. Second, Pearson's correlation between Depressive symptoms and Memory Valence was significantly negative $(r(48)=-0.30, p=0.04)$, indicating that higher scores on Depressive symptoms are associated with a more negative mother-related Memory Bias.

Finally, to test whether the effect of Trust remained significant when controlling for Depressive symptoms, the partial correlation between Memory Valence and Trust, controlled for Depressive symptoms, was calculated. This correlation remained marginally significantly positive $(r(47)=0.28, p=0.05)$. Conversely, the partial correlation between Memory Valence and Depressive symptoms, controlled for Trust, became non-significant $(r(47)=-0.22, p=0.14)$. 


\section{DISCUSSION}

The goal of the present study was to provide an improved test of the hypothesis that children who are less confident about maternal support have a more negative mother-related memory bias, compared to children who are more confident about maternal support. For this purpose, the current study investigated the relationship between attachment expectations and memory for positive and negative mother-referent adjectives, in a sample of school-aged children from the general population, using a well validated and widely used self-report measure of attachment, and controlling for the effect of depressive symptoms on memory bias. In line with the predictions, results revealed that children who are less confident about maternal support exhibited a more negative mother-related Memory Bias compared to more confident children. Furthermore this association between confidence in maternal support and memory bias remained marginally significant when controlling for depressive symptoms.

Demonstrating that children are most likely to process motherrelated information in congruence with their attachment expectancies is in line with previous findings (Rudolph et al., 1995), and confirms the assumption that recalling autobiographical attachment-related information leads to an attachmentexpectation congruent memory bias (Dykas \& Cassidy, 2011). This study contributes to the growing literature on attachmentrelated memory bias for three reasons.

First, children's attachment-related expectations were measured using a widely used and well-validated attachment questionnaire, making it possible to compare the findings with other research using this questionnaire. For example, the same questionnaire was used in a study on the attentional processing of mother (Bosmans et al., 2009). Combining the findings of both studies allows us to more decisively claim that self-reported attachment expectations modulate the cognitive processing of idiosyncratic attachment information, which might be import to understand the mechanisms guiding attachment behavior and leading to the development of psychopathology (Waters et al., 2013).

Second, this study is the first to simultaneously investigate this attachment-related memory bias' link with depressive symptoms. Although depressive symptoms were indeed related to this attachment-related memory bias, confidence in maternal support remained marginally significantly correlated with memory bias after controlling for the effect of depressive symptoms. Moreover, the results showed that the initial effect of depressive symptoms disappeared when taking into account confidence in maternal support, which means that there was no unique effect of depressive symptoms on this memory bias. This suggests that this attachment-related memory bias might indeed be a significant component of expectations regarding maternal support.

Third, this study showed that measuring memory biases in general population children allows to demonstrate that children recall information in congruence with their attachment expectations. We assume that this effect was masked in Lynch and Cicchetti's study (1998) with maltreated children, due to defensive exclusion processes aimed at avoiding the reactivation of severe psychological pain related to recalling traumatic interpersonal events. Confirming Bowlby's (1973) hypothesis in the current sample of non-clinical general population children, who had (most likely) not been physically and/or sexually abused by their attachment figures, suggest that defensive exclusion probably only occurs in severely traumatized children and is not part of the attachment-related memory bias of non-maltreated children.

\section{LIMITATIONS}

First, although the current study suggests that the approach to measure attachment-related memory biases is a promising strategy for future research on the importance of memory processes in the attachment system, more research is needed to confirm these findings using other measures of attachment expectations or using indicators that are conceptually linked to attachment, such as parenting measures. Indeed, attachment security is a complex construct in which both affective, cognitive and behavioral components have been considered central, and the current study only measured one specific, albeit central, component of the attachment security construct. Nevertheless, this study employed a well validated and widely used measure of explicit attachment expectations (Allen, in press; Armsden \& Greenberg, 1987), which has already been linked to other attachment-related information processing biases such as attention for motherrelated information (e.g., Bosmans et al., 2009) and with the Child Attachment Interview (Coffey, West, Somers, Mayfield \& Borelli, 2013).

Second, although this study showed a link between attachmentexpectations and mother-related memory bias, this link became marginally significant when depression was taken into account. Nevertheless, the finding that depressive symptoms was no longer related to attachment-related memory bias when taking into account attachment expectations, suggests that it is unlikely that this attachment-related memory bias merely reflects a depressionrelated memory bias. However, additional research is needed to investigate the relative contribution of attachment expectations and depressive symptoms in explaining mother-related memory bias. In addition, it might be interesting for future research to have a look at the potential role of this mother-related memory bias in the development of psychopathology. For instance, research could investigate, using longitudinal designs, whether this memory bias plays a role in explaining the link between insecure attachment expectations and the development of psychopathology.

Third, according to attachment theorists attachment-related memories are crucial for both the construction and consolidation of attachment expectations (Collins \& Read, 1994). However, the cross-sectional nature of the current study does not allow us to draw any causal conclusions regarding the link between trust and our attachment-related memory bias. Therefore, it would be interesting for future research to investigate this link using longitudinal designs.

\section{THEORETICAL AND CLINICAL IMPLICATIONS}

The current study's demonstrated memory bias might provide an interesting explanation for why attachment representations remain rather stable, and increasingly resistant to change (Bowlby, 1973, 1980; Weinfield, Sroufe \& Egeland, 2000). 
Insecurely attached children's tendency to recall less positive information regarding their mother might make them less sensitive to notice positive changes in parental behavior, which might decrease the likelihood that changes in parental behavior will alter their attachment representations. And although the current cross-sectional research design does not allow us to draw such causal conclusions, the results do suggest that the motherreferent encoding task might be a useful instrument to investigate this hypothesis in future longitudinal research.

A confirmation of this hypothesis in future research would also have important clinical implications. Results from a recent meta-analysis showed that adding parent management training to regular child-focused therapy has only a marginally significant additional effect (Dowell \& Ogles, 2010). Although this hypothesis needs further longitudinal investigation, the current findings suggest that, next to or in addition to changing parental behavior, uncertain children may benefit from a treatment that explicitly focuses on restoring the child's confidence in parental support. One promising new approach is Attachment Based Family Therapy, which successfully reduces adolescent depression through teaching parents to acknowledge and empathize with their children's disappointment regarding their parental support (ABFT; Diamond, Diamond \& Levy, 2013).

In sum, this study aimed to investigate the link between attachment expectations and autobiographical mother-related information in a general population sample. Results demonstrated a more negative mother-related memory bias in less securely attached children. Importantly, this effect could not be explained by depressive symptoms. This indicates that the current study's approach to investigate attachment-related memory bias is a promising strategy for further research on attachmentrelated memory bias. The results suggest that future research, especially with longitudinal designs, on attachment-related memory bias and its relationship with attachment representations is important, as it can increase our theoretical understanding of the development of attachment-related expectations and might help designing more effective intervention and prevention techniques.

\section{REFERENCES}

Ainsworth, M. D. S. (1973). The development of infant-mother attachment. In B. M. Caldwell \& H. N. Ricciuti (Eds.), Review of child development research (pp. 1-94). Chicago, IL: University of Chicago Press.

Ainsworth, M. D. S., Blehar, M. C., Waters, E. \& Wall, S. (1978). Patterns of attachment: A psychological study of the strange situation. Hillsdale: NJ: Erlbaum.

Alexander, K. W., O'Hara, K. D., Bortfeld, H. V., Anderson, S. J., Newton, E. K. \& Kraft, R. H. (2010). Memory for emotional experiences in the context of attachment and social interaction style. Cognitive Development, 25(4), 325-338.

Allen, J. P. (in press). Assessing attachment in adolescence. In E. Waters, B. Vaughn \& H. S. Waters (Eds.), Measuring attachment. New York: Guilford.

Allen, J. P., Porter, M. R., McFarland, F. C., McElhaney, K. B. \& Marsh, P. A. (2007). The relation of attachment security to adolescents' parental and peer relationships, depression, and externalizing behavior. Child Development, 78, 1222-1239.

Armsden, G. C. \& Greenberg, M. T. (1987). The Inventory of Parent and Peer Attachment - individual-differences and their relationship to psychological well-being in adolescence. Journal of Youth and Adolescence, 16, 427-454.
Beinstein Miller, J. \& Noirot, M. (1999). Attachment memories, models and information processing. Journal of Social and Personal Relationships, 16, 147-173.

Bosmans, G., Braet, C., Beyers, W., Van Leeuwen, K. \& Van Vlierberghe, L. (2011). Parents' power assertive discipline and internalizing problems in adolescents: The role of attachment. Parenting-Science and Practice, 11, 34-55.

Bosmans, G., Braet, C., De Coene, N. \& De Raedt, R. (2013). Trust in maternal support: children's attentional processing of mother and proximity seeking. Manuscript submitted for publication.

Bosmans, G., Braet, C., Koster, E. \& De Raedt, R. (2009). Attachment security and attentional breadth toward the attachment figure in middle childhood. Journal of Clinical Child and Adolescent Psychology, $38,872-882$.

Bosmans, G., Taghavi, M. R., Moradi, A. R., Yule, W. \& Dalgleish, K. (2008). [Psychometric evaluation of attachment questionnaires in a Flemish sample]. Unpublished raw data.

Bowlby, J. (1969). Attachment. London: Penguin Books.

Bowlby, J. (1973). Attachment and loss: Vol. 2. Separation: Anxiety and anger. New York: Basic.

Bowlby, J. (1980). Attachment and loss: Vol. 3. Loss, sadness, and depression. New York: Basic.

Brewer, W. F. \& Nakamura, G. V. (1984). The nature and functions of schema. In R. S. Wyer \& T. K. Srull (Eds.), Handbook of social cognition (pp. 119-160). Hillsdale, NJ: Erlbaum.

Coffey, J. K., West, J. L., Somers, J. A., Mayfield, C. \& Borelli, J. (2013). The child attachment interview: An empirical test of convergent and divergent validity. Paper presented at the Society of Research in Child Development (SRCD) Biennial Meeting, Seattle, WA.

Collins, N. L. \& Read, S. J. (1994). Cognitive representations of attachment: The structure and function of working models. In K. Bartholomew \& D. Perlman (Eds.), Advances in personal relationships (pp. 5390). London: Jessica Kingsley.

Craighead, W. E., Smucker, M. R., Craighead, L. W. \& Ilardi, S. S. (1998). Factor analysis of the Children's Depression Inventory in a community sample. Psychological Assessment, 10, 156-165.

Davidson, D. (1994). Recognition and recall of irrelevant and interruptive atypical actions in script-based stories. Journal of Memory and Language, 33, 757-775.

Diamond, G. S., Diamond, G. M. \& Levy, S. A. (2013). Attachmentbased family therapy for depressed adolescents. Washington, DC: American Psychological Association.

Dowell, K. A. \& Ogles, B. M. (2010). The effects of parent participation on child psychotherapy outcome: A meta-analytic review. Journal of Clinical Child and Adolescent Psychology, 39, 151-162.

Dubow, E. F. \& Ullman, D. G. (1989). Assessing social support in elementary-school children - the survey of childrens social support. Journal of Clinical Child Psychology, 18, 52-64.

Dykas, M. J. \& Cassidy, J. (2011). Attachment and the processing of social information across the life span: Theory and evidence. Psychological Bulletin, 137, 19-46.

Gullone, E. \& Robinson, K. (2005). The Inventory of Parent and Peer Attachment-Revised (IPPA-R) for children: A psychometric evaluation investigation. Clinical Psychology \& Psychotherapy, 12, $67-79$.

Hammen, C. \& Zupan, B. A. (1984). Self-schemas, depression, and the processing of personal information in children. Journal of Experimental Child Psychology, 37, 598-608.

Johnson, S. C., Dweck, C. S. \& Chen, F. S. (2007). Evidence for infants' internal working models of attachment. Psychological Science, 18, 501-502.

Kerns, K. A., Aspelmeier, J. E., Gentzler, A. L. \& Grabill, C. M. (2001). Parent-child attachment and monitoring in middle childhood. Journal of Family Psychology, 15, 69-81.

Kirsh, S. J. \& Cassidy, J. (1997). Preschoolers' attention to and memory for attachment-relevant information. Child Development, $68,1143-1153$.

Kovacs, M. (1992). Children's Depression Inventory. New York: Multi-Health Systems. 
Lynch, M. \& Cicchetti, D. (1998). Trauma, mental representation, and the organization of memory for mother-referent material. Development and Psychopathology, 10, 739-759.

Margolies, P. J. \& Weintraub, S. (1977). Revised 56-item CRPBI as a research instrument - reliability and factor structure. Journal of Clinical Psychology, 33, 472-476.

Neshat-Doost, H. T., Taghavi, M. R., Moradi, A. R., Yule, W. \& Dalgleish, T. (1998). Memory for emotional trait adjectives in clinically depressed youth. Journal of Abnormal Psychology, 107, 642-650.

Ridenour, T. A., Greenberg, M. T. \& Cook, E. T. (2006). Structure and validity of people in my life: A self-report measure of attachment in late childhood. Journal of Youth and Adolescence, 35, 1037-1053.

Rudolph, K. D., Hammen, C. \& Burge, D. (1995). Cognitive representations of self, family, and peers in school-age-children - links with social competence and sociometric status. Child Development, 66, 1385-1402.

Stangor, C. \& McMillan, D. (1992). Memory for expectancy - congruent and expectancy -incongruent information. A review of the social and social developmental literatures. Psychological Bulletin, 111, 42-61.

Taylor, L. \& Ingram, R. E. (1999). Cognitive reactivity and depressotypic information processing in children of depressed mothers. Journal of Abnormal Psychology, 108, 202-210.

Timbremont, B. \& Braet, C. (2002). Children's Depression Inventory: Nederlandstalige versie (Dutch version). Lisse: Swets \& Zeitlinger.
Timbremont, B. \& Braet, C. (2004). Cognitive vulnerability in remitted depressed children and adolescents. Behaviour Research and Therapy, 42, 423-437.

Timbremont, B. \& Braet, C. (2005). Selective information-processing in depressed children and adolescents: Is there a difference in processing of self-referent and other-referent information? Behaviour Change, 22, 143-155.

van Ijzendoorn, M. H. (1995). Adult attachment representations, parental responsiveness, and infant attachment - a metaanalysis on the predictive-validity of the adult attachment interview. Psychological Bulletin, 117, 387-403

Waters, T. E. A., Brockmeyer, S. L. \& Crowell, J. A. (2013). AAI Coherence predicts caregiving and care seeking behaviour: Secure base script knowledge helps explain why. Attachment \& Human Development, 15, 316-331.

Weinfield, N. S., Sroufe, L. A. \& Egeland, B. (2000). Attachment from infancy to early adulthood in a high-risk sample: Continuity, discontinuity, and their correlates. Child Development, 71, 695-702.

Wisco, B. E. (2009). Depressive cognition: Self-reference and depth of processing. Clinical Psychology Review, 29, 382-392.

Received 3 May 2013, accepted 13 March 2014 